\title{
Superbackscattering Antenna Arrays
}

\author{
Iñigo Liberal, Member, IEEE, Iñigo Ederra, Ramón Gonzalo, Member, IEEE, and \\ Richard W. Ziolkowski, Fellow, IEEE
}

\begin{abstract}
This article discusses the theory, design and practical implementation of superbackscattering antenna arrays. In analogy with Uzkov's maximal directivity theorem, it is demonstrated that the maximal backscattering cross-section, normalized to the wavelength squared, of a linear array of $N$ isotropic scatterers whose separation tends to zero is $N^{2}(N+1)^{2} /(4 \pi)$. This analytical result is validated via numerical optimization of the excitation coefficients, and the same procedure is utilized to assess the maximal backscattering of arrays of electric Hertzian dipoles (EHDs). It is found that electrically small arrays of two and three EHDs can enhance the backscattering by factors of 6.22 and 22.01, respectively, with respect to the maximum value generated by a single element. In addition, physical realizations of arrays featuring comparable enhancement factors can be straightforwardly designed by using a simple procedure inspired by Yagi-Uda antenna concepts. The practical implementations of such arrays based on copper wires and printed circuit technologies is also addressed.
\end{abstract}

Index Terms-Backscattering, electromagnetic scattering, superdirectivity, antenna theory, electrically small antennas.

\section{INTRODUCTION}

Enhancing the backscattering cross-section of a receiving antenna is of natural interest for passive RFID [1], batteryfree wireless [2] or ambient backscatter [3] antenna systems. In addition, highly backscattering antennas are exceptionally visible objects, which could be utilized as contrast agents in imaging and characterization systems [4]. Moreover, random mixtures of these antennas, even when very diluted, are able to extinguish the propagation of electromagnetic fields. Hence, they also meet applications as electromagnetic shields and radar countermeasures. Furthermore, the larger the backscattering cross-section, the larger the flow of kinetic momentum directed against the propagation of the incident field. Therefore, momentum conservation indicates that the pushing forces induced by propagating fields are augmented in highly backscattering antennas [5]-[7]. As a result, they are also of great interest for electromagnetic manipulation systems. Consequently, one finds that antennas exhibiting unusually large backscattering cross-sections have important impacts on a wide range of communication, identification,

I. Liberal, I. Ederra and R. Gonzalo are with the Electrical and Electronic Engineering Department, Universidad Pública de Navarra, Pamplona, Spain, e-mail: inigo.liberal@unavarra.es

R. W. Ziolkowski is with the Department of Electrical and Computer Engineering, University of Arizona, Tucson, AZ, 85721 USA, email:ziolkowski@ece.arizona.edu

This work was supported in part by the Spanish Ministry of Science and Innovation, Dirección General de Investigación y Gestión del Plan Nacional de I+D+I, Subdirección General de Proyectos de Investigación, Project No. CSD2008-00066 and TEC2013-47753-C3-1-R, and by NSF contract number ECCS-1126572. I.L. acknowledges financial support from a FPI scholarship of UPNA.

Manuscript received Oct. XX, 2014; revised Feb. YY, 2015 ... sensing, monitoring, and even electromagnetic manipulation technologies.

It is a well known fact that an antenna can scatter more power than the total amount incident onto its geometrical area [8], [9]. However, the scattering produced by a passive receiving antenna is limited by fundamental reasons. For instance, causality limits the amount of achievable scattering over a certain bandwidth [10]-[12]; and, even for purely timeharmonic fields, the amount of scattering is limited by energy conservation [13]-[17]. Additional restrictions apply to the scattering directed towards specific directions. In particular, an upper bound on the backscattering cross-section (normalized to the wavelength squared) is given by [13]

$$
\sigma_{\mathrm{b}}\left(-\widehat{\mathbf{k}}_{i}\right) \leq \frac{1}{\pi} D_{\text {scat }}\left(-\widehat{\mathbf{k}}_{i}\right) D_{\text {scat }}\left(\widehat{\mathbf{k}}_{i}\right)
$$

where $D_{\text {scat }}\left(\widehat{\mathbf{k}}_{i}\right)$ and $D_{\text {scat }}\left(-\widehat{\mathbf{k}}_{i}\right)$ are the scattering directivities in the forward, $\widehat{\mathbf{k}}_{i}$, and backward, $-\widehat{\mathbf{k}}_{i}$, directions [13], [14]. The bound (1) is a direct consequence of the optical theorem (energy conservation) [18].

The bound (1) indicates that the backscattering cross-section can be enhanced by using antennas with highly directive scattering patterns that point to both the forward and backward directions. Following this philosophy, one classic approach to engineer the directivity of antennas is the use of antenna arrays [19]. In essence, antenna arrays benefit from the coherent radiation emitted by several antennas, leading to a larger radiation aperture than that of the individual elements. In this manner, most antenna arrays consist of structures whose size is on the order of several wavelengths. However, it was pointed out by Uzkov [20] that the maximum endfire directivity of a linear array actually increases as the separation between the elements tends to zero. This result opened up the possibility of designing electrically small superdirective antenna arrays [21]-[23]. Admittedly, the development of efficient electrically small superdirective arrays is hindered by strict fabrication tolerances, narrow bandwidths and low radiation efficiencies. Nevertheless, experimental demonstrations of efficient and superdirective two-element endfire arrays have been presented [21]-[23]. There have also been successful implementations based on printed circuit technologies [24], though these are inevitably associated with lower radiation efficiencies because of the intrinsic material losses.

Both the upper bound (1) and previous results on superdirective antenna arrays [20]-[24] encouraged us to believe that the design of superbackscattering antenna arrays should also be possible. In order to confirm this outcome conclusively, we particularize the bound (1) first to investigate the maximal backscattering cross-sections of linear antenna arrays. We then address the design of antenna arrays approaching those 


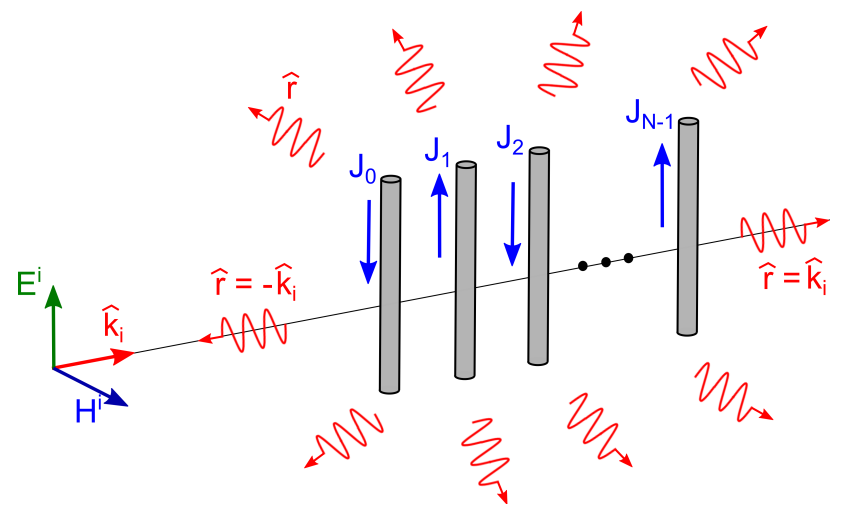

Fig. 1. Sketch of the scattering problem: an antenna array is illuminated by a plane-wave propagating along the $\widehat{\mathbf{k}}_{i}$ direction. The interaction of the incident field with the array results in the excitation of currents in the antenna elements and the subsequent radiation of the scattered fields in all $\widehat{\mathbf{r}}$ directions.

theoretical limits. In contrast with classical superdirective antenna arrays [20]-[24], the proposed superbackscattering antenna arrays are illuminated by an external wave, and are engineered to exhibit an unusually large backscattering crosssection by focusing the scattered field in both the forward and backward directions.

The remainder of the paper is organized as follows. First, Section II introduces the geometry of the problem and basic antenna array notation. This notation is employed in Section III to analytically and numerically investigate the upper bounds of the backscattering cross-section of linear antenna arrays. The design of two-element antenna arrays approaching those theoretical limits is addressed next in Section IV. Then, in Section $\mathrm{V}$, the performance of practical implementations based on thick copper wires and printed elements is analyzed. To finalize, conclusions and a discussion of the results are gathered in Section VI. Time-harmonic $e^{j \omega t}$ field expressions are assumed throughout and are omitted hereafter. All crosssection and power quantities are written as their time-averaged embodiments.

\section{GeOMETRY AND NOTATIONS}

Fig. 1 schematically depicts the geometry of the problem: an antenna array is illuminated by a plane-wave propagating along the $\widehat{\mathbf{k}}_{i}$ direction. Due to the interactions between the incident field $\left(\mathbf{E}^{i}, \mathbf{H}^{i}\right)$ and the antenna array, conduction and polarization currents are excited in the antenna elements. The fields radiated by these sources constitute the scattered (or re-radiated) fields, $\left(\mathbf{E}^{s}, \mathbf{H}^{s}\right)$, which propagate away from the antenna in all $\widehat{\mathbf{r}}$ directions. Particular cases of interest are the forward $\left(\widehat{\mathbf{r}}=\widehat{\mathbf{k}}_{i}\right)$ and backward $\left(\widehat{\mathbf{r}}=-\widehat{\mathbf{k}}_{i}\right)$ directions. Subsequently, the total field is equal to the sum of the incident and scattered fields $\mathbf{E}=\mathbf{E}^{i}+\mathbf{E}^{s}$ and $\mathbf{H}=\mathbf{H}^{i}+\mathbf{H}^{s}$.

As does the incident field, the set of conduction/polarization currents and scattered fields satisfy Maxwell equations. This equivalent radiation process can be mathematically formulated by invoking either the volume equivalence or induction theorems [25], [26]. Therefore, the scattering directivity can be described with the same formalism used for the radiation directivity in conventional antenna arrays. Consequently, the antenna notation is adopted here to investigate the upper bound on the scattering (1) for specific array configurations. In particular, in the far-zone, the scattered field reduces to a spherical wave with electric field $\mathbf{E}^{s}=\mathbf{f}(\widehat{\mathbf{r}}) \exp \left(-j k_{0} \widehat{\mathbf{r}} \cdot \mathbf{r}\right) /\left(k_{0} r\right)$, where $\mathbf{f}(\widehat{\mathbf{r}})$ is the far-field scattering pattern along the observation direction $\widehat{\mathbf{r}}$. For an array composed of $N$ receiving antennas/scatterers, $\mathbf{f}(\widehat{\mathbf{r}})$ can be written as follows [19].

$$
\mathbf{f}(\widehat{\mathbf{r}})=\sum_{n=0}^{N-1} a_{n} \mathbf{f}_{n}(\widehat{\mathbf{r}}) e^{j k_{0} \widehat{\mathbf{r}} \cdot \mathbf{r}_{n}}
$$

where $a_{n}, \mathbf{r}_{n}$ and $\mathbf{f}_{n}(\widehat{\mathbf{r}})$ are the excitation coefficient, position and far-field scattering pattern of the $n^{t h}$ antenna element/scatterer.

The backscattering cross-section, i.e., the radar cross section in the direction of the source, is defined and normalized to the wavelength squared as follows [14], [27]

$$
\sigma_{\mathrm{b}}\left(-\widehat{\mathbf{k}}_{i}\right)=\frac{1}{\pi}\left|\frac{\mathbf{f}\left(-\widehat{\mathbf{k}}_{i}\right)}{E_{0}}\right|^{2}
$$

where $E_{0}$ is the amplitude of the incident field. Similarly, the far-field scattering directivity is defined as follows [14], [28]

$$
D_{\text {scat }}(\widehat{\mathbf{r}})=\frac{4 \pi|\mathbf{f}(\widehat{\mathbf{r}})|^{2}}{\int_{0}^{\pi} \int_{0}^{2 \pi}\left|\mathbf{f}\left(\widehat{\mathbf{r}}^{\prime}\right)\right|^{2} \sin \theta^{\prime} d \theta^{\prime} d \phi^{\prime}}
$$

where the integration is carried out over the coordinate angles associated with all $\widehat{\mathbf{r}}^{\prime}$ directions. For the scattering application under consideration it is convenient to rewrite the directivity in the following manner: [21]

$$
D_{\text {scat }}(\widehat{\mathbf{r}})=\frac{\sum_{n=0}^{N-1} \sum_{m=0}^{N-1} \mathbf{A}_{n} \cdot \mathbf{A}_{m}^{*} e^{j k_{0} \widehat{\mathbf{r}} \cdot\left(\mathbf{r}_{n}-\mathbf{r}_{m}\right)}}{\sum_{n=0}^{N-1} \sum_{m=0}^{N-1} \mathbf{A}_{n} \cdot \mathbf{A}_{m}^{*} h_{n m}(\widehat{\mathbf{r}})}
$$

with $\mathbf{A}_{n}=a_{n} \mathbf{f}_{n}(\widehat{\mathbf{r}})$ and $h_{n m}(\widehat{\mathbf{r}})$ defined as follows

$$
h_{n m}(\widehat{\mathbf{r}})=\frac{H_{n m}}{\mathbf{f}_{n}(\widehat{\mathbf{r}}) \cdot \mathbf{f}_{m}^{*}(\widehat{\mathbf{r}})},
$$

with

$$
\begin{aligned}
& \quad H_{n m}=\frac{1}{4 \pi} \int_{0}^{\pi} \int_{0}^{2 \pi} \mathbf{f}_{n}\left(\widehat{\mathbf{r}}^{\prime}\right) \cdot \mathbf{f}_{m}^{*}\left(\widehat{\mathbf{r}}^{\prime}\right) e^{j \widehat{\mathbf{r}}^{\prime} \cdot \mathbf{u}_{n m}} \sin \theta^{\prime} d \theta^{\prime} d \phi^{\prime} \\
& \text { where } \mathbf{u}_{n m}=k_{0}\left(\mathbf{r}_{n}-\mathbf{r}_{m}\right), u_{n m}=\left|\mathbf{u}_{n m}\right|
\end{aligned}
$$

\section{THEORY}

Uzkov's theorem states that the maximal endfire directivity of a linear array of $N$ isotropic radiators equals $N^{2}$ as their separation approaches zero [20]. In fact, this is the maximal directivity of any 3D array composed of $N$ isotropic radiators [29]. These results suggested that electrically small superdirective arrays were possible [21]. Consequently, it should be equally possible to enhance the forward-backward scattering directivity product, thus opening up the possibility of designing superbackscattering antenna arrays. This is in fact the case. As we derive in Appendix A, the normalized backscattering cross-section of a linear array of $N$ isotropic scatterers illuminated along the array axis (see Fig.2(a)), 
(a)

(b)
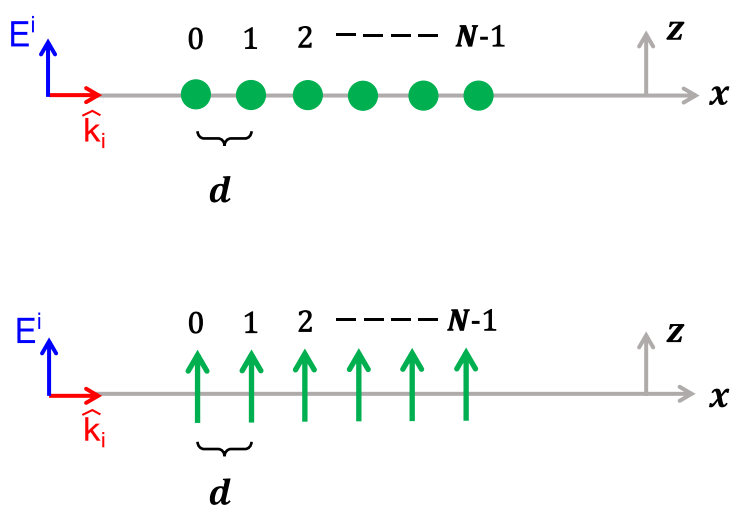

Fig. 2. Sketch of linear arrays of $N$ elements with constant separation $d$ illuminated by a plane wave propagating along the array axis. (a) Isotropic and (b) electric Hertzian dipole cases.

whose separation distance $d$ approaches zero, has the upper bound:

$$
\sigma_{b}\left(-\widehat{\mathbf{k}}_{i}\right) \leq \frac{1}{4 \pi} N^{2}(N+1)^{2}
$$

Eq. (8) is the backscattering counterpart to Uzkov's theorem. It establishes that the backscattering from a set of isotropic radiators can be greatly enhanced by the use of electrically small arrays, even when very few elements are employed. This analytical result can be numerically tested by writing the directivity as in Eq. (5), and numerically optimizing the excitation coefficients $\mathbf{A}_{n}$. Instead of directly maximizing the upper bound (1), we elect to optimize the forward-backward directivity by minimizing its inverse. Specifically, the nonlinear minimization problem is solved by using the NelderMead simplex algorithm [30] as implemented in Matlabß. It is also useful in the case of isotropic radiators to note that the radiation pattern can be considered scalar $(f(\widehat{\mathbf{r}})=1)$ and the integral in Eq. (7) then simplifies to [21]

$$
h_{n m}(\widehat{\mathbf{r}})=H_{n m}=\frac{\sin \left(u_{n m}\right)}{u_{n m}}
$$

Fig. 3(a) represents the backscattering cross-section, as a function of element separation distance, for optimized linear arrays of two and three isotropic elements. It is found that the backscattering bound increases as the separation of the elements tends to zero. It is important to emphasize that in end fire antenna arrays, the area transverse to the direction of scattering/radiation does not increase along with the array size. Therefore, one cannot simply apply the common assumption that the directivity will increase by making the array size larger. Contrarily, we prove that, in analogy with superdirective effects radiation in antenna arrays [20], [21], the best backscattering results are theoretically obtained with increasingly smaller arrays. In fact, the limiting $d \rightarrow 0$ cases converge to Eq. (8), validating the analytical derivation.

It is worth noting, as derived in [31]-[33], that Eq. (8) also corresponds to the maximal bistatic cross-section of an acoustic system which excites $\mathrm{N}$ spherical harmonics efciently and that it is identical to Eq. (36) in [31]. Consequently, it can also

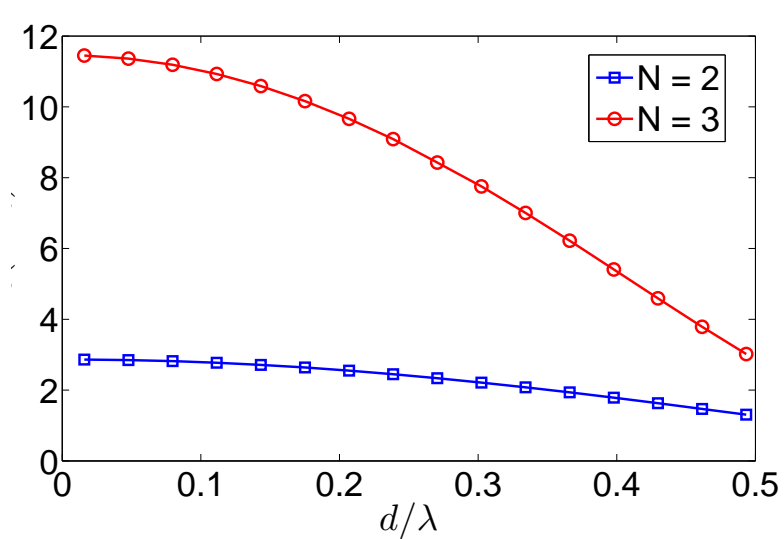

(a)

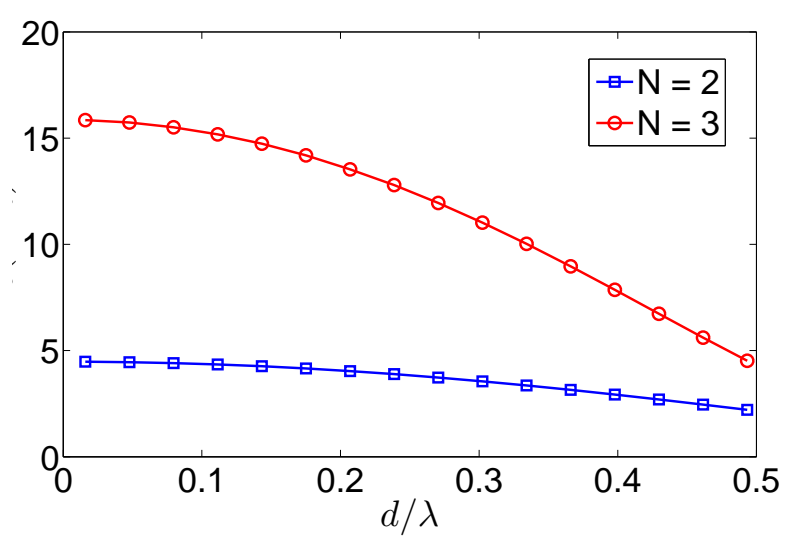

(b)

Fig. 3. Optimized backscattering cross-section, as a function of the element separation distance, for linear arrays of two and three (a) isotropic and (b) EHD elements.

be demonstrated that the same result holds for the backscattering cross-section, i.e., the backscattering cross-section is itself a maximum of the bistatic cross-section. In addition, this implies that the backscattering performance of a linear array of $N$ isotropic radiators is equivalent to an acoustic system which excites $N$ spherical harmonics. However, it must be emphasized that these outcomes are particular cases of isotropic radiators, e.g., of acoustic systems. In contrast, the maximal backscattering cross-section associated with electromagnetic fields is always smaller than the maximal bistatic cross-section, which takes place in the forward direction [13]. This difference between electromagnetic and acoustic systems arises from the fact that there are no isotropic radiators of electromagnetic fields. On the other hand, electromagnetic radiators allow the possibility of combining electric and magnetic multipoles to increase the directivity in the forward direction, an outcome that cannot take place in acoustic systems.

Thus, while isotropic scatterers are an interesting and analytically tractable approximation to the problem, they might be too crude of an approximation of real antenna elements. Fortunately, a similar numerical procedure can be applied to the case of electric Hertzian dipole (EHD) elements. To this end, we consider the element radiation patterns: $\mathbf{f}(\widehat{\mathbf{r}})=\widehat{\boldsymbol{\theta}} \sin \theta$, for (without any loss of generality) EHD elements located 
along the $\mathrm{x}$-axis and oriented along $\widehat{\mathbf{z}}$ (cf., Fig. 2(b)). In this manner, $h_{n m}(\widehat{\mathbf{r}})=H_{n m} / \sin ^{2} \theta$, and $H_{n m}$ can be written in closed form as: [21]

$$
H_{n m}=\frac{\sin \left(u_{n m}\right)}{u_{n m}}\left(1-\frac{1}{u_{n m}^{2}}\right)+\frac{\cos \left(u_{n m}\right)}{u_{n m}^{2}}
$$

Fig. 3(b) represents the optimized backscattering crosssection for linear arrays of two and three EHD radiators as a function of the element separation distance. As with isotropic scatterers, the backscattering increases as the separation decreases. However, note that the backscattering values for the limiting $d \rightarrow 0$ cases (4.48 and 15.85 for arrays of two and three scatterers, respectively) are larger than those obtained for isotropic scatterers. This is a natural consequence of the larger directivity of the individual EHD elements.

Note that the backscattering bound of a single EHD (which has equal forward and backward directivity) is $1.5^{2} / \pi \simeq 0.72$. Therefore, the results reported in Fig. 3(b) reveal that the use of two and three element antenna arrays can potentially lead to backscattering enhancement factors of 4.48/0.72 $=6.22$ and $15.85 / 0.72=22.01$, respectively, with respect to the maximum value generated by a single element. We also note that the maximal backscattering cross-section of a system efficiently exciting $N$ multipoles is $\left(N^{2}+2 N\right)^{2} /(4 \pi)$ [13], which predicts backscattering peak values of $\left(2^{2}+2 \times 2\right)^{2} /(4 \pi) \simeq 5.1$ and $\left(3^{2}+2 \times 3\right)^{2} /(4 \pi) \simeq 19$, respectively, for the efficient excitation of two and three multipoles. These backscattering peak values are larger than those obtained for arrays of two and three electric Hertzian dipoles. This outcome indicates, in contrast to the acoustic case, that a linear array of $N$ electric Hertzian dipoles cannot produce a backscattering value that achieves the $N$ spherical harmonic limit.

Nevertheless, even though it is a theoretical abstraction, an EHD can be considered to be a good approximation to any electrically small dipole antenna. Therefore, the theoretical results presented here indicate that the backscattering of electrically small dipole antennas can be greatly enhanced by arranging them in electrically small arrays. The design of these antenna systems is addressed next.

\section{Design Procedure}

Knowing that superbackscattering antenna arrays are theoretically possible, the next natural question is how to identify and design their physical realizations. Unfortunately, the theoretical description of electrically small antenna arrays is a very challenging task. The complexity arises from the intricate reactive interactions between near-field coupled antenna elements. Therefore, we will focus here on providing simple design guidelines that enable approaching the theoretical results through numerical considerations. The theoretical analysis of the reactive interactions between the array elements is left for future efforts. Following previously reported experiences with superdirective antenna arrays [21]-[24], the designs are restricted here to two-element antenna arrays to simplify the discussion.

We first outline the design process before delving into the details of the numerical simulations. The bound (1) indicates that the backscattering is maximized in structures with a balanced forward-backward scattering pattern. Following this philosophy, we first consider a two-element array of identical elements, and examine its scattering pattern. The subsequent radiation pattern might be unbalanced initially, i.e., the directivity might be higher either in the forward or backward direction. Then, this asymmetry is corrected by using YagiUda antenna concepts. Specifically, if the directivity is larger in the forward (backward) direction, then the first (second) element (along the direction of propagation) is scaled down in size until the pattern is balanced. From a Yagi-Uda perspective, the shorter element becomes a director for the longer element, whereas the longer element becomes a reflector of the shorter element. This methodology enables strengthening the scattering pattern towards the forward or backward directions.

This design procedure is validated through the use of numerical simulations with the commercial software ANSYS HFSS. To this end, we start with the numerical analysis of the individual antenna elements. In order to simplify the discussion, we initially consider antenna elements constructed from perfect electric conductor (PEC) materials. The impact of losses will be addressed later in Section V. The HFSS model and geometry of the single antenna element is schematically depicted in Fig. 4. This top-hat loaded dipole element is selected as the antenna element due to its relatively small size $\left(r_{\text {out }} / \lambda=0.1\right.$ at $\left.2 \mathrm{GHz}\right)$, EHD-like scattering pattern, and familiarity from several metamaterial-inspired near-field resonant parasitic antenna designs, e.g., [34]. This antenna acts purely as a scatterer; there is no port and/or load connected to it. In practice, the resonance frequency shifts that are introduced by any sensor and/or chip connected to it can be compensated by adjusting the geometry of the dipole and/or by including near-field parasitic elements.

The backscattering cross-section of this antenna element is presented in Fig. 5. The backscattering spectrum is characterized by a resonance at approximately $2 \mathrm{GHz}$, featuring a peak value of 0.81 . This value is slightly larger than the backscattering bound for an EHD: $1.5^{2} / \pi \simeq 0.72$, due to the non-zero physical length of the element. The scattering directivity patterns in the E- (solid) and H- (dashed) planes are depicted in the inset of Fig. 5. This figure confirms that the element is characterized by an EHD-like scattering pattern. Furthermore, the forward and backward scattering directivities are both approximately equal to 1.6. Thus the peak value in the main figure is consistent with the upper bound (1), i.e, $1.6^{2} / \pi \simeq 0.815$. This exemplifies how physical realizations of dipole antenna elements can actually beat theoretical bounds based of conceptual EHDs.

Next we investigate the performance of a two-element array with identical elements separated by a distance $d$, which is depicted in Fig. 6. The backscattering spectrum of this array as a function of the element separation is given in Fig. 7. It can be concluded that as the separation distance decreases, the backscattering spectra are characterized by increasingly narrower and higher peak resonances. It is also apparent from Fig. 7 that the peak backscattering is enhanced four to five times with respect to the single element (cf. Fig. 5). However, this performance is still below the bounds studied in Section III. This fact is more clearly appreciated in Fig. 8, which 


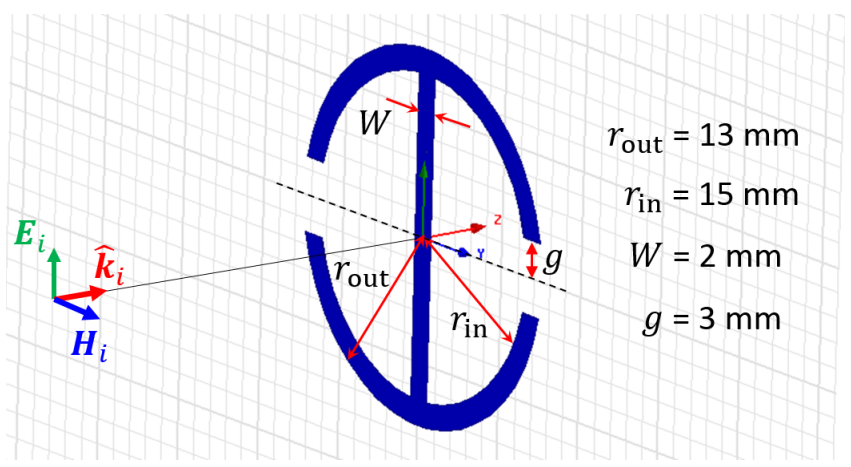

Fig. 4. Top-hat loaded dipole geometry: HFSS model along with its variables and optimized dimensions.

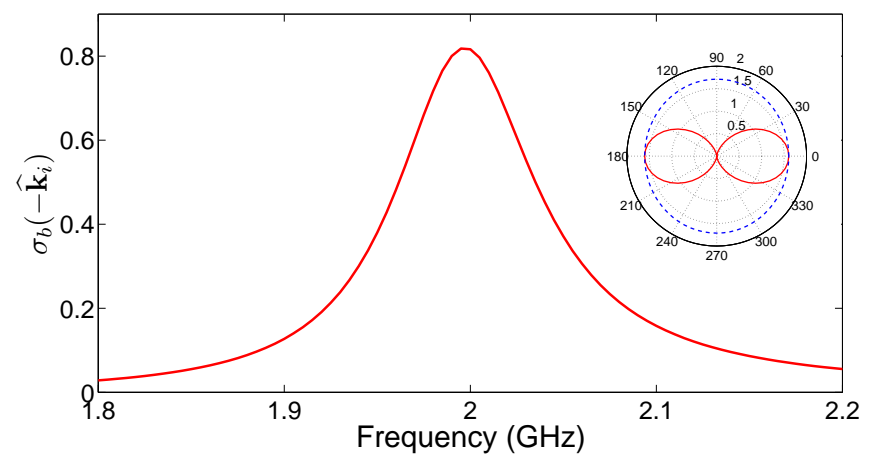

Fig. 5. Backscattering cross-section of the top-hat loaded dipole. Inset: Scattering directivity patterns in the E- (solid) and $\mathrm{H}$ - (dashed) planes at the resonance frequency: $1.995 \mathrm{GHz}$.

represents the peak backscattering cross-section as a function of element separation. The upper bounds for arrays of isotropic and EHD elements are also included for comparison purposes. This figure reveals that, while the individual elements are more directive than a single EHD dipole, the backscattering of the array of identical elements is still below the bound for an array of EHD dipoles.

In order to get a deeper insight into the limitations of arrays with identical elements, Fig. 9 depicts the scattering directivity patterns in the $\mathrm{E}$ - (solid) and $\mathrm{H}$ - (dashed) planes at the frequencies for which the peak backscattering of this two element top-hat loaded dipole array for separation distances of $d=2,4,6,8$ and $10 \mathrm{~mm}$. This set of figures shows that arrays of identical elements tend to direct the scattering towards the

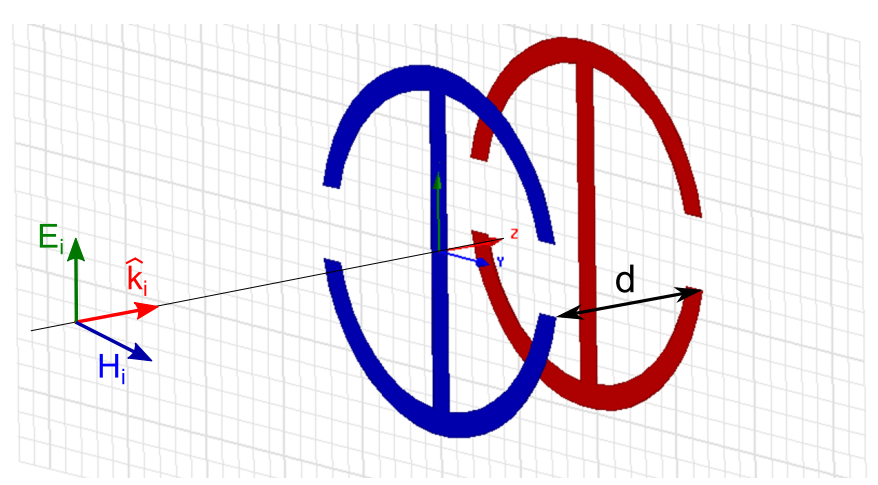

Fig. 6. HFSS model of the two-element linear array of top-hat loaded dipoles.

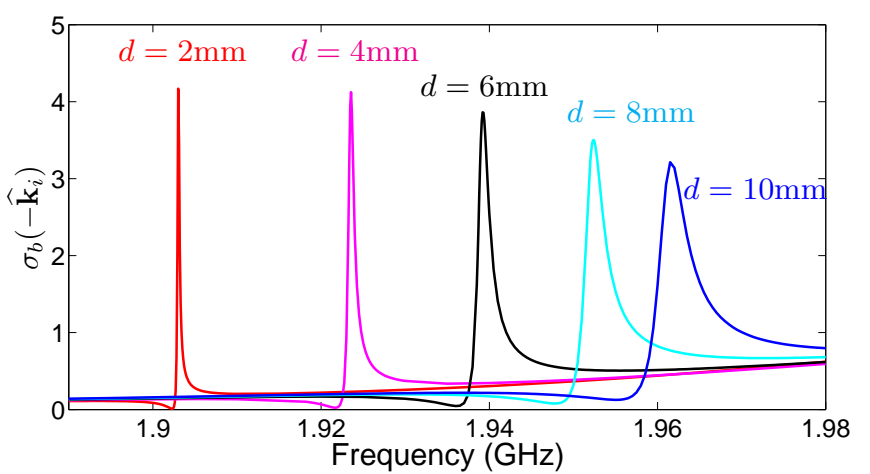

Fig. 7. Backscattering cross-sections of two-element linear arrays of identical top-hat loaded dipoles for separation distances ranging from 2 to $10 \mathrm{~mm}$.

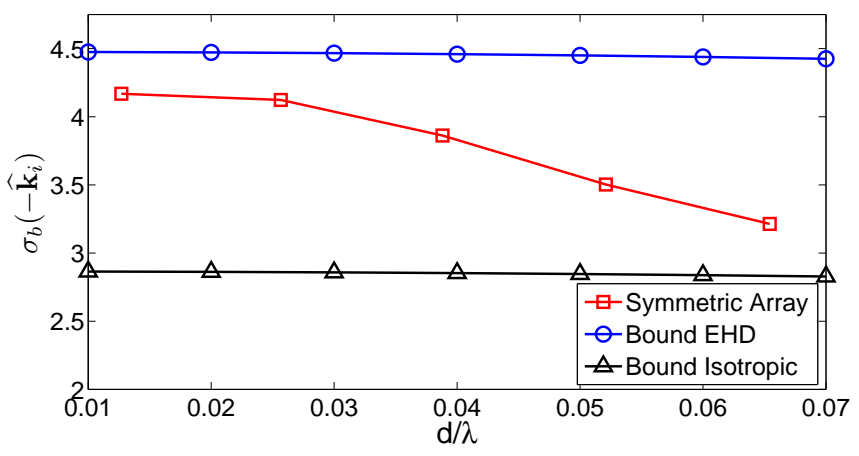

Fig. 8. Peak backscattering cross-section, as a function of element separation (normalized to the wavelength), for a two-element linear array of identical tophat loaded dipoles. For comparison, the upper bounds for arrays of isotropic and EHD elements is provided.

forward direction. However, this tendency is mitigated as the separation distance between the array elements decreases. The higher backscattering peaks in Fig. 7 are consistent with the bound (1); they are associated with the patterns which feature a more forward-backward balanced scattering in Fig. 9. We further note that no additional lobes emerge in the scattering pattern as the separation between the elements shrinks. This is a clear indicator that the excitation of higher order modes in the single antenna elements remains weak, and thus the dipolar approximation assumed in Section III is still reasonably accurate.

Fortunately, the backscattering cross-section can be optimized at a specific separation distance by following the aforementioned guidelines based on Yagi-Uda antennas. In particular, this is achieved by scaling down in size the first element encountered by the incident wave, i.e, the left element in Fig. 6.

It is theoretically possible to attain increasingly larger enhancement factors as the separation between the elements decreases (see Fig.3(b)). However, from a practical point of view, the narrowing of the resonances observed in Fig.7 makes the array more difficult and time consuming to optimize numerically. Consequently, taking into account fabrication tolerance issues, the separation between the elements is set at $10 \mathrm{~mm}$ as a reasonable value to test and to demonstrate that one could obtain interesting backscattering enhancements even with an easy to manufacture value. Fig. 10 represents 

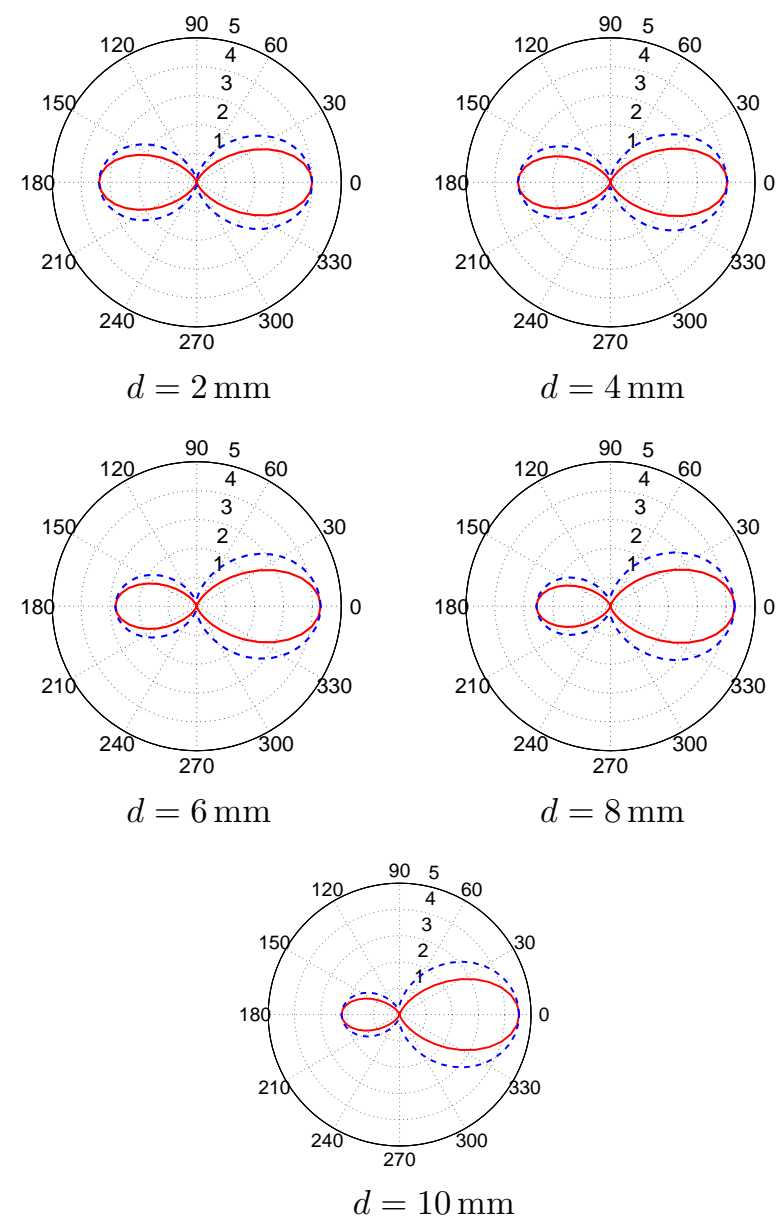

Fig. 9. Scattering directivity patterns, in the E- (solid) and H- (dashed) planes, at the resonance frequency of the two-element linear arrays of identical top-hat loaded dipoles having the separation distances: $d=2 \mathrm{~mm}$ (at $1.903 \mathrm{GHz}$ ), $d=4 \mathrm{~mm}$ (at $1.924 \mathrm{GHz}), d=6 \mathrm{~mm}$ (at $1.940 \mathrm{GHz}$ ), $d=8 \mathrm{~mm}$ (at $1.952 \mathrm{GHz}$ ) and $d=10 \mathrm{~mm}($ at $1.962 \mathrm{GHz}$ ).

the peak backscattering cross-section and the front-to-back scattering ratio (FBSR) as a function of the scaling factor, for a separation distance of $d=10 \mathrm{~mm}$. Following the philosophy of Yagi-Uda antennas, the symmetry of the two elements is broken by the size scaling of the first element. The FBSR monotonically decreases as the scaling factor increases. In contrast, the backscattering peak is maximized when the FBSR equals one. The maximal obtained backscattering peak equals 4.71, which is slightly larger that the 4.48 value obtained for the EHD arrays in Section III. This effect is again caused by the larger directivity of the top-hat loaded dipoles with respect to the idealized EHDs. Note that the enhancement factor with respect to the single antenna element $(4.71 / 0.81 \simeq 5.81)$ is very similar to that obtained in the theoretical analysis of the EHD case.

In conclusion, superbackscattering antenna arrays can be designed in a straightforward manner by using a simple design procedure inspired by Yagi-Uda antenna configurations. In order to complete the description, Fig. 11 depicts the scattering directivity patterns, in the E- (solid) and $\mathrm{H}$ - (dashed) planes, at the backscattering peak for the scaling factors: $s=0.999$, $s=0.993$ and $s=0.987$. The patterns ratify the observa-

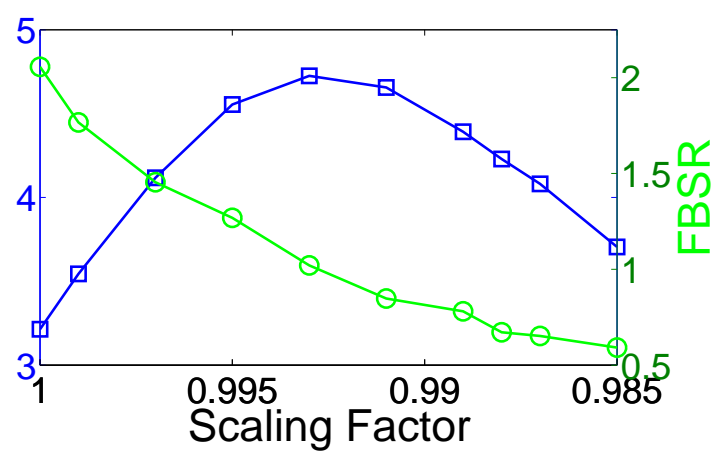

Fig. 10. Peak backscattering cross-section and front-to-back scattering ratio of a two-element linear array of top-hat loaded dipoles with an element separation distance of $d=10 \mathrm{~mm}$, as a function of the scaling factor of the first (in the direction of propagation) element.
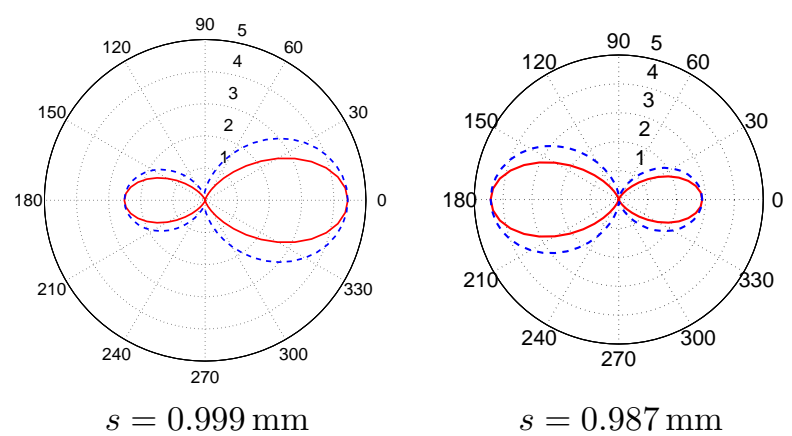

$s=0.987 \mathrm{~mm}$

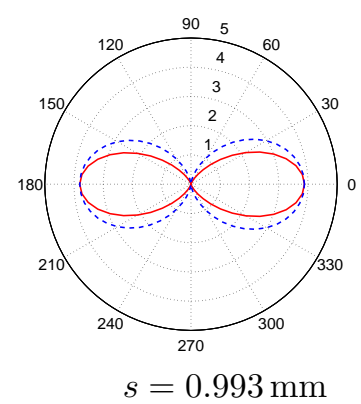

Fig. 11. Scattering directivity patterns, in the E- (solid) and H- (dashed) planes, at the backscattering peak frequency $(f \simeq 1.96 \mathrm{GHz})$ of the twoelement linear array of top-hat loaded dipoles when the separation distance is $d=10 \mathrm{~mm}$, and where the first element has been reduced in size by the scaling factors: $s=0.999, s=0.993$ and $s=0.987$.

tion that a transition from an emphasis on the forward to backward scattering will occur as the scaling factor decreases. Consistently, the best backscattering performance in Fig. 10 (scaling factor $s=0.993$ ) corresponds to a highly directive pattern, with maximal directivity equal to 3.89 , and with beams pointing equally in both the forward and backward directions.

\section{Practical Implementations}

While the superbackscattering antenna arrays were straightforwardly designed using ideal lossless materials, we cannot overlook the fact that, just like with their superdirective counterparts, they consist of very high-Q resonators. Therefore, they are bound to be penalized by the inclusion of losses when using realistic materials. Despite this fact, electrically small superdirective antenna arrays were experimentally validated 
successfully in [21], [22], [24]. This encourages us to believe than the same will be true for superbackscattering antenna arrays. Two examples aimed at assessing the practicality and viability of superbackscattering antenna arrays are presented.

First, the efficient superdirective arrays in [21], [22] were designed using copper wires of $1.6 \mathrm{~mm}$ diameter. Similarly, our first example is based on copper strips of $1.6 \mathrm{~mm}$ thickness. The geometry, schematically depicted in the inset of Fig. 12(a), is the same as the one in Figs. 4 and 6, and the array was designed by using the procedure introduced in Section IV. Consequently, the separation between elements was again fixed at $d=10 \mathrm{~mm}$, and the scaling factor was optimized. The optimal value was determined to be $s=0.99$. The simulated performance of the designed array is presented in Fig. 12. Fig. 12(a) represents the backscattering cross-section of the array as a function of the frequency. For comparison purposes, the figure also includes the backscattering crosssection of a single antenna element, as well as the optimized implementation of the lossless two-element array. The peak backscattering values for the lossless and lossy twoelement arrays are 4.71 and 3.23 , respectively. On the other hand, the backscattering peak value of the single antenna element is 0.79 . Therefore, it is concluded that although the backscattering is attenuated by dissipation damping, the twoelement array with realistic materials nevertheless enables a backscattering enhancement by a factor of 4.1 with respect to the single antenna element. Moreover, it also is apparent from Fig. 12 that the backscattering cross-section is actually larger than that of the single antenna element over the entire studied frequency range.

For the sake of completeness, the scattering directivity patterns of the single antenna element and antenna array (both lossy) are depicted in Figs. 12(b) and 12(c), respectively. The single antenna element results are clearly characterized as an EHD-like scattering pattern, as expected from theory. On the other hand, as in the lossless case, the optimized realistic superbackscattering array generates a highly directive pattern simultaneously pointing towards the forward and backward directions.

Although using thick copper is arguably the best strategy to enhance the efficiency (i.e., to minimize the conduction losses) of electrically small antennas, many applications demand the use of antennas fabricated with printed circuit technologies. Therefore, our second example consists of a superbackscattering antenna array implemented with a commercial substrate. Specifically, we use a 2-oz (70 $\mu$ m thick copper), 5-mil (0.127 $\mathrm{mm})$ thick substrate, Rogers Duroid 5880 board material (characterized by the material parameters: $\varepsilon_{r}=2.2, \mu_{r}=1$, $\tan \delta=0.0009)$. The array geometry, schematically depicted in the inset of Fig. 13(a), is the same as in the one in Figs. 4 and 6 , again with the separation distance $d=10 \mathrm{~mm}$. The array was designed by using the procedure introduced in Section IV. The optimal scaling factor was determined to be $s=0.99$ via a numerical parameter study. The simulated scattering performances of the optimized-design array, its lossless counterpart, and the corresponding single scattering element are compared in Fig. 13. First, the backscattering cross-sections are depicted in Fig. 13(a). The peak backscattering values for the lossless

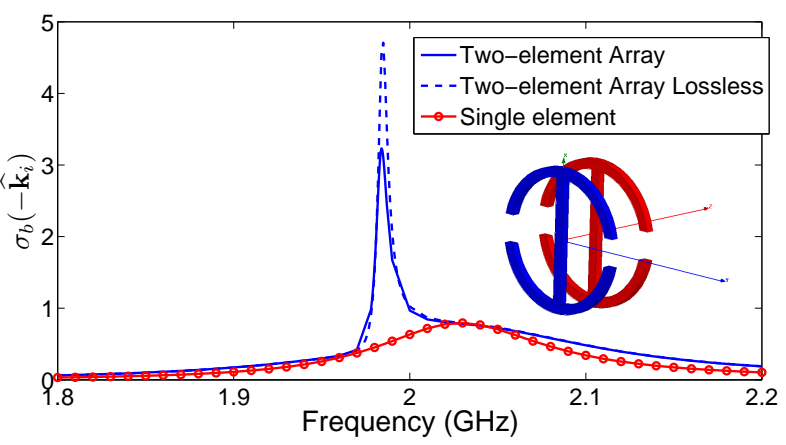

(a)

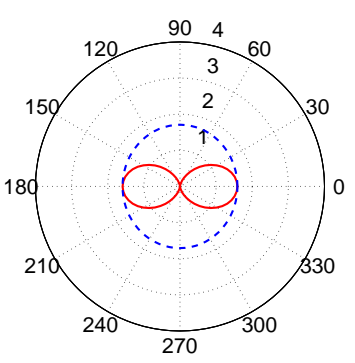

(b)

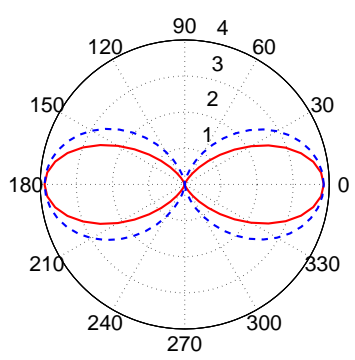

(c)
Fig. 12. (a) HFSS-predicted values of the backscattering cross-section for the two-element linear array of top-hat loaded dipoles which are separated by a distance $d=10 \mathrm{~mm}$, constructed with coppers strips of $1.6 \mathrm{~mm}$ thickness, and the first (left) element is scaled by a factor $s=0.99$ with respect to the second (right) element. For comparison purposes, the figure also includes the backscattering cross-section of an ideal lossless array and a single antenna element. Inset: Sketch of the geometry. Scattering directivity patterns in the E(solid) and $\mathrm{H}$ - (dashed) planes for the (b) single element and (c) two-element array.

and lossy two-element arrays are 4.45 and 1.89 , respectively. On the other hand, the peak value of the single antenna element is only 0.79 . These numerical values further exemplify how dissipation damping has a more severe impact on printed circuit elements than on those constructed with thick copper wires. Nevertheless, on the bright side, the peak backscattering value is enhanced by a factor of 2.39 with respect to the single antenna element. In addition, the backscattering values of the array remain larger than those of the single element at most of the studied frequencies. Therefore, this example suggests that the superbackscattering antenna arrays could also be successfully implemented with printed circuit technologies. To finalize the comparisons, Figs. 13(b) and 13(c) depict the scattering directivity patterns of the single element and the optimized printed two-element antenna array, respectively. The pattern of the single antenna element has the expected dipolar form; the two-element array exhibits the anticipated highly directive and balanced patterns.

\section{CONCLUSions}

The theory, design and practical implementation of superbackscattering antenna arrays has been discussed. The backscattering counterpart to Uzkov's theorem has been derived, i.e., it has been demonstrated that the maximal backscattering cross section, normalized to the wavelength squared, of a linear array of $N$ equally spaced isotropic scatterers converges to $N^{2}(N+1)^{2} /(4 \pi)$ as the separation distance 


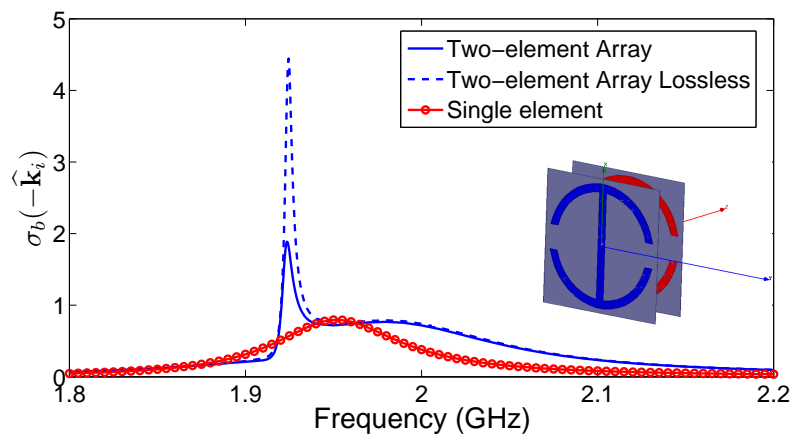

(a)

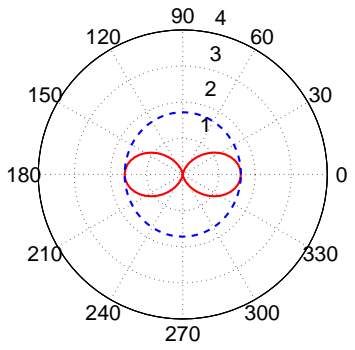

(b)

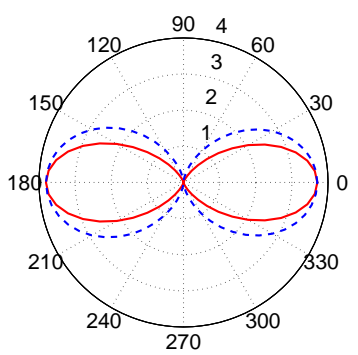

(c)
Fig. 13. (a) HFSS-predicted values of the backscattering cross-section for a two-element linear array of top-hat loaded dipoles constructed with coppers strips printed with a $70 \mu \mathrm{m}$ thickness over a RO5880 substrate that is $5 \mathrm{mil}$ $(0.127 \mathrm{~mm})$ thick. The first (left) element is scaled by a factor $s=0.99$ with respect to the second (right) element. The separation distance between them equals $d=10 \mathrm{~mm}$. For comparison purposes, the figure also includes the backscattering cross-section of an ideal lossless array and a single antenna element. Inset: Sketch of the geometry. Scattering directivity patterns in the E(solid) and H- (dashed) planes for the (b) single element and (c) two-element array.

approaches zero. This analytical result has been validated by using numerical optimization techniques. The same analytical and numerical techniques were utilized to obtain the corresponding results for arrays of EHDs. These analyses have revealed that, even when very few elements are employed, electrically small antenna arrays theoretically lead to large enhancements of the backscattering cross-section. Specifically, it is found that arrays of two and three EHDs elements can potentially lead to backscattering enhancement factors of 6.22 and 22.01, respectively, with respect to the maximum value generated by a single element. Moreover, it was demonstrated that arrays of more complicated elements which feature comparable enhancement factors can be straightforwardly designed using a simple procedure inspired by Yagi-Uda antenna concepts.

However, in analogy with superdirective antenna arrays, the superbackscattering antenna arrays are high-Q resonators. Consequently, it was anticipated and then demonstrated that their performance characteristics are penalized by losses that arise naturally within the materials used to fabricate them. Despite this fact, it was found that two element arrays of tophat loaded dipoles implemented as thick copper wires and as printed elements lead to backscattering enhancement factors of 4.1 and 2.39, respectively, with reasonable choices of their design parameters. We emphasize than even better performance results could be obtained with more sophisticated designs, including for example, strategies to reduce dissipation losses, antenna elements with larger radiation resistances and/or optimized separation distances. These results thus encourage us to believe that superbackscattering antenna arrays can indeed be implemented in practice, meeting applications in a wide range of communication, identification, sensing, monitoring, and even electromagnetic manipulation technologies.

\section{APPENDIX A \\ DERIVATION OF EQ. (8)}

In order to prove Eq. (8) let us consider a linear array of isotropic $\left(f_{n}(\widehat{\mathbf{r}})=1\right)$ radiators, located along the $\mathrm{Z}$-axis with constant separation $d$ (i.e., $\mathbf{r}_{n}=\widehat{\mathbf{z}}(n-1) d$ ). In such a case, the far-field radiation pattern of the array can be written as follows

$$
f(\widehat{\mathbf{r}})=\sum_{n=0}^{N-1} a_{n} e^{j n k_{0} d \cos \theta}
$$

As demonstrated by Uzkov [20], the radiation pattern (11) can be rewritten in the $k_{0} d \rightarrow 0$ limit as a series of Legendre polynomials:

$$
\lim _{k_{0} d \rightarrow 0} \sum_{n=0}^{N-1} a_{n} e^{j n k_{0} d \cos \theta}=\sum_{n=0}^{N-1} c_{n} P_{n}(\cos \theta)
$$

The reader is referred to [20] for the original discussion. However, the author provides scarce details of the proof. A more intuitive explanation can be found in the appendix of [21]. For the interest of our readers, an independent derivation of Uzkov's theorem is included as supplementary material.

The decomposition (12) is particularly useful since we can make use of the orthogonality property of Legendre polynomials [26]

$$
\int_{0}^{\pi} P_{n}(\cos \theta) P_{m}(\cos \theta) \sin \theta d \theta=\delta_{n m} \frac{2}{2 n+1}
$$

and their special values [26]

$$
P_{n}(1)=1, \quad P_{n}(-1)=(-1)^{n}
$$

in order to write the forward-backward directivity product as follows

$$
D_{\text {scat }}(\widehat{\mathbf{z}}) D_{\text {scat }}(-\widehat{\mathbf{z}})=\frac{\left|\sum_{n=0}^{N-1} c_{n}\right|^{2}\left|\sum_{n=0}^{N-1}(-1)^{n} c_{n}\right|^{2}}{\left(\sum_{n=0}^{N-1} \frac{1}{2 n+1}\left|c_{n}\right|^{2}\right)^{2}}
$$

Let us define the auxiliary complex variables

$$
\begin{aligned}
C_{e} & =\sum_{n \text { even }} c_{n}=\left|C_{e}\right| e^{j \varphi_{e}} \\
C_{o} & =\sum_{n \text { odd }} c_{n}=\left|C_{o}\right| e^{j \varphi_{o}}
\end{aligned}
$$

where $\{n$ even $\}=0,2,4, \ldots$ and $\{n$ odd $\}=1,3,5, \ldots$ The numerator of (15) can written in terms of $C_{e}$ and $C_{o}$ as follows

$$
\left|\sum_{n=0}^{N-1} c_{n}\right|^{2}\left|\sum_{n=0}^{N-1}(-1)^{n} c_{n}\right|^{2}=\left|C_{e}+C_{o}\right|^{2}\left|C_{e}-C_{o}\right|^{2}
$$




$$
\begin{gathered}
=\left(\left|C_{e}\right|^{2}+\left|C_{o}\right|^{2}\right)^{2}-4\left|C_{e}\right|^{2}\left|C_{o}\right|^{2} \cos ^{2}\left(\varphi_{e}-\varphi_{o}\right) \\
\leq\left(\left|C_{e}\right|^{2}+\left|C_{o}\right|^{2}\right)^{2}
\end{gathered}
$$

In this manner, the product $D_{\text {scat }}(\widehat{\mathbf{z}}) D_{\text {scat }}(-\widehat{\mathbf{z}})$ can be upper bounded as follows

$$
\begin{aligned}
& D_{\text {scat }}(\widehat{\mathbf{z}}) D_{\text {scat }}(-\widehat{\mathbf{z}}) \leq \frac{\left(\left|C_{e}\right|^{2}+\left|C_{o}\right|^{2}\right)^{2}}{\left(\sum_{n=0}^{N-1} \frac{1}{2 n+1}\left|c_{n}\right|^{2}\right)^{2}}= \\
\leq & {\left[\frac{\left(\sum_{n \text { even }}\left|c_{n}\right|\right)^{2}+\left(\sum_{n \text { odd }}\left|c_{n}\right|\right)^{2}}{\sum_{n=0}^{N-1} \frac{1}{2 n+1}\left|c_{n}\right|^{2}}\right]^{2}=D_{\text {aux }}^{2} }
\end{aligned}
$$

Note that the first derivative

$$
\begin{gathered}
\frac{d}{d\left|c_{n}\right|}\left\{D_{\text {aux }}\right\}= \\
\begin{cases}\frac{2}{\sum_{n=0}^{N-1} \frac{1}{2 n+1}\left|c_{n}\right|^{2}}\left(S_{e}-D_{\text {aux }} \frac{\left|c_{n}\right|}{2 n+1}\right) & n \text { even } \\
\frac{2}{\sum_{n=0}^{N-1} \frac{1}{2 n+1}\left|c_{n}\right|^{2}}\left(S_{O}-D_{\text {aux }} \frac{\left|c_{n}\right|}{2 n+1}\right) & n \text { odd }\end{cases}
\end{gathered}
$$

where

$$
S_{e}=\sum_{n \text { even }}\left|c_{n}\right|, \quad S_{o}=\sum_{n \text { odd }}\left|c_{n}\right|
$$

Consequently, the solution to the maximization problem: $d / d\left|c_{n}\right|\left\{D_{\text {aux }}\right\}=0$ is given by

$$
\left|c_{n}\right|_{\text {max }}= \begin{cases}(2 n+1) \frac{S_{e}}{D_{\text {aux }}} & n \text { even } \\ (2 n+1) \frac{S_{o}}{D_{\text {aux }}} & n \text { odd }\end{cases}
$$

Adding the maximized even and odd $\left|c_{n}\right|_{\text {max }}$ terms separately, one obtains the expressions

$$
\left\{\begin{array}{l}
S_{e, \max }=\sum_{n \text { even }}(2 n+1) \frac{S_{e, \max }}{D_{\text {aux }, \max }} \\
S_{o, \max }=\sum_{n \text { odd }}(2 n+1) \frac{S_{o, \max }}{D_{\text {aux }, \max }}
\end{array}\right.
$$

Therefore, the maximum is either

$$
D_{\text {aux }, \max }=\sum_{n \text { even }}(2 n+1)=\left(2 N_{e}+1\right)\left(N_{e}+1\right)
$$

or

$$
D_{\text {aux }, \max }=\sum_{n \text { odd }}(2 n+1)=\left(2 N_{o}+1\right) N_{o}
$$

where $N_{e}$ and $N_{o}$ are, respectively, the total number of even and odd numbers in the set $\{1,2, \ldots, N-1\}$. Note that it can be readily checked (e.g., by taking $N=1$ ) that

$$
\sum_{n \text { even }}(2 n+1) \neq \sum_{n \text { odd }}(2 n+1)
$$

Therefore, there are two solutions of the maximization problem which are given by $D_{\text {aux }}=S_{e}, S_{o}=0$ and $D_{\text {aux }}=$ $S_{o}, S_{e}=0$.

Physically, this means that one cannot make an efficient use of both even and odd modes, with respect to the associated Legendre polynomial decomposition (12), to enhance the forward-backward directivity product. On the contrary, one has to chose between the excitation of either even or odd modes to get the optimal response for a given number of radiators $N$. For instance, if $N$ is an even number, then $N_{e}=N / 2-1$ and $N_{o}=N / 2$, Inserting these into Eqs. (24) and (25) gives:

$$
\begin{aligned}
& \sum_{n \text { even }}(2 n+1)=\frac{N(N-1)}{2} \\
& \sum_{n \text { odd }}(2 n+1)=\frac{N(N+1)}{2}
\end{aligned}
$$

On the other hand, if $N$ is an odd number, then $N_{e}=$ $(N-1) / 2$ and $N_{o}=(N-1) / 2$. These lead lead to corresponding sums

$$
\begin{aligned}
& \sum_{n \text { even }}(2 n+1)=\frac{N(N+1)}{2} \\
& \sum_{n \text { odd }}(2 n+1)=\frac{N(N-1)}{2}
\end{aligned}
$$

Thus, if $N$ is an even (odd) number, then the optimal response is obtained when properly exciting all odd (even) modes in the Legendre polynomial decomposition, while inhibiting the even (odd) modes. Nevertheless, the same maximum value is reached in either case. It is given by

$$
D_{\text {aux }, \text { max }}=\frac{N(N+1)}{2}
$$

Consequently, the forward-backward directivity product has the upper bound:

$$
D_{\text {scat }}(\widehat{\mathbf{z}}) D_{\text {scat }}(-\widehat{\mathbf{z}}) \leq D_{\text {aux }}^{2} \leq \frac{N^{2}(N+1)^{2}}{4}
$$

Finally, it follows from Eqs. (32) and (1) that the backscattering cross-section of a linear array of $N$ elements whose scattering patterns are isotropic and whose separation approaches zero has the upper bound:

$$
\sigma_{b}(-\widehat{\mathbf{z}}) \leq \frac{1}{\pi} D_{\text {scat }}(\widehat{\mathbf{z}}) D_{\text {scat }}(-\widehat{\mathbf{z}}) \leq \frac{1}{4 \pi} N^{2}(N+1)^{2}
$$

\section{REFERENCES}

[1] K. Finkenzeller, RFID Handbook, 3rd ed. New York: Wiley, 2010.

[2] M. Philipose, J. R. Smith, B. Jiang, A. Mamishev, S. Roy, and K. Sundara-Rajan, "Battery-free wireless identification and sensing," IEEE Pervasive Computing, vol. 4, no. 1, pp. 37-45, 2005.

[3] V. Liu, A. Parks, V. Talla, S. Gollakota, D. Wetherall, and J. R. Smith, "Ambient backscatter: wireless communication out of thin air," in Proceedings of the ACM SIGCOMM, 2013, pp. 39-50.

[4] L. Tong, Q. Wei, A. Wei, and J.-X. Cheng, "Gold nanorods as contrast agents for biological imaging: optical properties, surface conjugation and photothermal effects," Photochemistry and Photobiology, vol. 85, no. 1, pp. 21-32, 2009.

[5] M. Nieto-Vesperinas, R. Gómez-Medina, and J. J. Sáenz, "Anglesuppressed scattering and optical forces on submicrometer dielectric particles," J. Opt. Soc. Am. A, vol. 28, no. 1, pp. 54-60, 2011.

[6] J. J. Sáenz, "Optical forces: laser tractor beams," Nature Photonics, vol. 5, no. 9, pp. 514-515, 2011.

[7] J. Chen, J. Ng, Z. Lin, and C. T. Chan, "Optical pulling force," Nature Photonics, vol. 5, no. 9, pp. 531-534, Jul. 2011.

[8] C. F. Bohren, "How can a particle absorb more than the light incident on it?" Am. J. Phys., vol. 51, no. 4, pp. 323-327, Apr. 1982.

[9] C. F. Bohren and D. R. Huffman, Absorption and Scattering of Light by Small Particles. New York: John Wiley \& Sons, 2008.

[10] C. Sohl, M. Gustafsson, and G. Kristensson, "Physical limitations on broadband scattering by heterogeneous obstacles," J. Phys. A, vol. 40, no. $36,11165,2007$. 
[11] — , "Physical limitations on metamaterials: restrictions on scattering and absorption over a frequency interval," J. Phys. D, vol. 40, no. 22 , pp. 7146-7151, 2007.

[12] C. Sohl, C. Larsson, M. Gustafsson, and G. Kristensson, "A scattering and absorption identity for metamaterials: Experimental results and comparison with theory," J. Appl. Phys., vol. 103, no. 5, pp. 4906-4910, 2008.

[13] I. Liberal, I. Ederra, R. Gonzalo, and R. W. Ziolkowski, "Upper bounds on scattering processes and metamaterial-inspired structures that reach them," IEEE Trans. Antennas Propag., vol. 62, no. 12, pp. 6344-6353, 2014.

[14] J. B. Andersen and A. Frandsen, "Absorption efficiency of receiving antennas," IEEE Trans. Antennas Propag., vol. 53, no. 9, pp. $2843-$ 2849, Sept. 2005.

[15] I. Liberal, Y. Radi, R. Gonzalo, I. Ederra, S. A. Tretyakov, and R. W. Ziolkowski, "Least upper bounds of the power extracted and scattered by bi-anisotropic particles," IEEE Trans. Antennas Propag., vol. 62, no. 9, pp. 4726-4735, Sept. 2014.

[16] I. Liberal, I. Ederra, R. Gonzalo, and R. W. Ziolkowski, "A multipolar analysis of near-field absorption and scattering processes," IEEE Trans. Antennas Propag., vol. 61, no. 10, pp. 5184-5199, Oct. 2013.

[17] I. Liberal and R. W. Ziolkowski, "Analytical and equivalent circuit models to elucidate power balance in scattering problems," IEEE Trans. Antennas Propag., vol. 61, no. 5, pp. 2714-2726, May 2013.

[18] R. G. Newton, "Optical theorem and beyond," Am. J. Phys., vol. 44, no. 7, pp. 639-642, 1976.

[19] C. A. Balanis, Antenna Theory: Analysis and Design, 3rd ed. New York: John Wiley \& Sons, 2012.

[20] A. I. Uzkov, "An approach to the problem of optimum directive antennae design," Comptes Rendus (Doklady) de l'Academie des Sciences de l'URSS, vol. 53, pp. 35-38, 1946.

[21] E. E. Altshuler, T. H. O’Donnell, A. D. Yaghjian, and S. R. Best, "A monopole superdirective array," IEEE Trans. Antennas Propag., vol. 53, no. 8, pp. 2653-2661, 2005.

[22] A. D. Yaghjian, T. H. O'Donnell, E. E. Altshuler, and S. R. Best, "Electrically small supergain end-fire arrays," Radio Sci., vol. 43, no. 3, RS3002, 2008.

[23] A. D. Yaghjian, "Increasing the supergain of electrically small antennas using metamaterials," in EuCAP 2009. 3rd European Conf. Antennas Propag., Berlin, Germany, 2009, pp. 858-860.

[24] T. Kokkinos and A. P. Feresidis, "Electrically small superdirective endfire arrays of metamaterial-inspired low-profile monopoles," IEEE Antennas Wireless Propag. Lett., vol. 11, pp. 568-571, 2012.

[25] I. Liberal, I. Ederra, R. Gonzalo, and R. W. Ziolkowski, "Induction theorem analysis of resonant nanoparticles: Design of a Huygens source nanoparticle laser," Phys. Rev. Applied, vol. 1, no. 4, 044002, May 2014.

[26] R. F. Harrington, Time-Harmonic Electromagnetic Fields. New York: McGraw-Hill, 1961

[27] C. A. Balanis, Advanced Engineering Electromagnetics. New York: John Wiley \& Sons, 2012.

[28] I. Liberal, I. Ederra, R. Gonzalo, and R. W. Ziolkowski, "Circuit and multipolar approaches to investigate the balance of powers in $2 \mathrm{D}$ scattering problems," PIER, vol. 55, pp. 277-301, 2013.

[29] A. T. Parsons, "Maximum directivity proof for three-dimensional arrays," J. Acoustical Soc. Am., vol. 82, no. 1, pp. 179-182, 1987.

[30] J. C. Lagarias, J. A. Reeds, M. H. Wright, and P. E. Wright, "Convergence properties of the nelder-mead simplex method in low dimensions," SIAM J. Optimization, vol. 9, no. 1, pp. 112-147, 1998.

[31] A. D. Yaghjian, "Sampling criteria for resonant antennas and scatterers," J. Appl. Phys., vol. 79, no. 10, pp. 7474-7482, May 1996.

[32] Y. Liu, "Spherical array superdirective beamforming based on spherical harmonic decomposition of the soundfield," in OCEANS, 2012-Yeosu. IEEE, 2012, pp. 1-5.

[33] M. A. Gerzon, "Maximum directivity factor of nth-order transducers," J. Acoustical Soc. Am., vol. 60, no. 1, pp. 278-280, 1976.

[34] P. Jin and R. W. Ziolkowski, "Metamaterial-inspired, electrically small Huygens sources," IEEE Antennas Wireless Propag. Lett., vol. 9, pp. 501-505, 2010

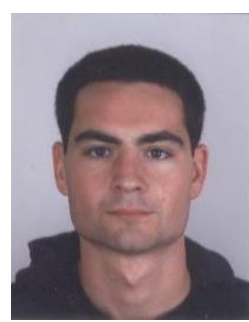

Iñigo Liberal received the Engineer's (2009), M.Sc. (2010) and Ph. D. (2013), magna cum laude, degrees in Telecommunication Engineering from the Public University of Navarra (UPNA), Spain. He was a visiting student at Delft University of Technology (Delft, The Netherlands), and a visiting researcher at Aalto University (Helsinki, Finland), the University of Arizona (Tucson, USA) and the University of Pennsylvania (Philadelphia, USA).

He currently is a postdoctoral fellow at the Antenna Group, Department of Electrical and Electronic Engineering, Public University of Navarra (UPNA), Spain. His main research interests are in the fields of scattering and antenna theory, with emphasis on electromagnetic manipulation and trapping, electrically small antennas and artificial electromagnetic materials.

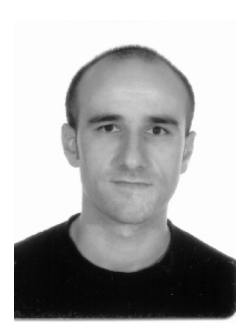

Iñigo Ederra was born in Isaba, Navarra, Spain in 1972. He received the Ingeniero de Telecomunicación and $\mathrm{Ph} . \mathrm{D}$. degrees from the Universidad Pública de Navarra, Pamplona, Spain, in 1996 and 2004, respectively.

In 1997, he joined the Microwave and Millimetre Wave Group, Universidad Pública de Navarra. From 1999 to 2000 he was with the European Space Research and Technology Centre (ESTEC), ESA, Noordwijk, The Netherlands, where he was working on Electromagnetic Bandgap materials and their applications in the field of antennas. Since 2001 he is with the Antenna Group, Universidad Pública de Navarra. From June to October 2002 he was visitor scientist at the Rutherford Appleton Laboratory, Chilton, Didcot, UK, participating in the Startiger project.

He was co-recipient of the LAPC 2006 and IWAT 2007 best paper award. His research interests are in the field of Electromagnetic Bandgap materials and Metamaterials and their applications in microwave and millimetre wave components and antennas.

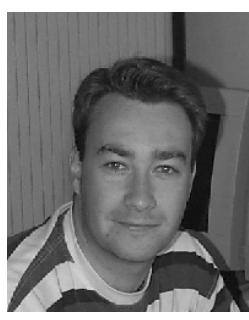

Ramón Gonzalo was born in Logroño, La Rioja. He received the M.Sc. and the $\mathrm{Ph}$. D. degree in Ingeniero de Telecomunicación both with honors, from the Public University of Navarra (UPNa), Spain.

Since October 1995 he is with the Antennas Group at the Electrical and Electronic Engineering Department in UPNa where he currently is Full Professor. From September 1997 to December 1998, he was joined as Research Fellow to the Antenna Section in ESA-ESTEC where he was involved in the modelling and design of electromagnetic crystal devices at microwave and millimetre wave frequencies. He has been involved in more than 25 research project, European, and National level acting as co-ordinator in several of them. In particular he has been co-ordinator of 5 projects funded by the European Space Agency, 3 projects in the framework of the European Commission and several of them funded by the Spanish Minister of Science. He has more than 70 journal publications in peer review magazines and 120 conference papers related to his research lines. From January 2006 until April 2008 he was acting as sub-director of the Engineering Faculty and from April 2008 to December 2010 as Head of Electrical and Electronic Engineering Department.

He was co-recipient of the LAPC 2006, LAPC 2007 and IWAT 2007 best paper award. His current area of research is in the field of Terahertz technologies, sub-systems and devices, Electromagnetic Band Gap technology with emphasis on space antenna applications, design of waveguide transmission lines and corrugated horn antennas. 
This is the author's version of an article that has been published in this journal. Changes were made to this version by the publisher prior to publication.

The final version of record is available at http://dx.doi.org/10.1109/TAP.2015.2410787

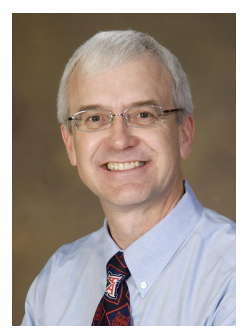

Richard W. Ziolkowski (M'87-SM'91-F'94) received his Sc.B. degree in physics, magna cum laude with honors, from Brown University (1974), and his M.S. (1975) and Ph.D. (1980) degrees in physics from the University of Illinois at UrbanaChampaign. $\mathrm{He}$ is the Litton Industries John M. Leonis Distinguished Professor in the Department of Electrical and Computer Engineering at the University of Arizona. He is also a Professor in the College of Optical Sciences at the University of Arizona. He is a Distinguished Adjunct Professor to King Abdulaziz University (KAU), Jeddah, Saudi Arabia. He was awarded an Honorary Doctorate, Doctor Technish Honoris Causa, from the Technical University of Denmark (DTU) in 2012. He was the Computational Electronics and Electromagnetics Thrust Area Leader in the Engineering Research Division at the Lawrence Livermore National Laboratory before joining the University of Arizona in 1990. He is the 2014-2015 Australian DSTO Fulbright Distinguished Chair in Advanced Science and Technology.

Prof. Ziolkowski is a Fellow of both the Institute of Electrical and Electronics Engineers (IEEE) and the Optical Society of America (OSA) He served as the President of the IEEE Antennas and Propagation Society in 2005. He is also actively involved with the URSI, OSA and SPIE professional societies. He and Prof. Nader Engheta, University of Pennsylvania, are CoEditors of the best-selling 2006 IEEE-Wiley book, Metamaterials: Physics and Engineering Explorations. 